\title{
Stability and vibrations control of a stepped beam using piezoelectric actuation
}

\author{
Krzysztof Kuliński ${ }^{1}$, Jacek Przybylski ${ }^{1{ }^{*}}$ \\ ${ }^{1}$ Częstochowa University of Technology, Institute of Mechanics and Machine Design Fundamentals, \\ Częstochowa, Dabrowskiego 69 street, Poland, EU
}

\begin{abstract}
The objects of this studies are the stability and transversal vibrations of the system composed of three segments, where in the centre part of the system two piezoelectric patches are perfectly bonded to the top and bottom surface of the host beam. The system is kinematically loaded as a result of prescribed displacement of one or both end supports. For the analysis purposes three different beam end supports have been taken into consideration, which prevent longitudinal displacements i.e. clamped-clamped, clamped-pinned and pinned-pinned. This type of beam loading not only affect its natural vibration frequencies but also the system's stability. By introducing the electric field to the piezo patches, depending on its vector direction, in-plane stretching or compressive residual force may be induced. Presented results show that piezo actuation can significantly modify both the critical buckling force and the vibration frequency.
\end{abstract}

Keywords: stability, vibrations, stepped beam, piezo actuation

\section{Introduction}

Rapid technological and research development have a crucial effect on mechanical properties of newly produced materials, allowing thereby application of beams and columns with higher slenderness at comparable level of buckling capacity. From the mechanical point of view, in case of slender systems the examination of only stability criterion is insufficient, thus additional element failure due to the vibrations should have been studied. It is well known that elements exposed to significant vibrations should be protected from the resonance phenomenon. There are variety of ways to modify fundamental systems stability and vibrations in order to prevent the resonance such as bonding another material to the host beam/column, changing the type of support or supports, adding another supporting point etc. In this paper additional material bonding method to a host beam is presented, applying piezoelectric patches, which are also called in literature as "smart materials". These materials allow not only change the beam/column structural parameters as axial and bending stiffness, which result in increased buckling capacity, but may be also used as passive modification of system vibrations frequency and furtherly the buckling capacity via the piezo actuation. Piezo actuation is the reverse piezoelectric effect, where electric field is applied to the material in order to change its dimensions. According to

\footnotetext{
*Corresponding author: jacek.pr@imipkm.pcz.pl

Reviewers: Vladimír Dekýš, Tomáš Lack
} 
above additional pretension axial force can be generated in a beam/column, affecting its stability and vibrations.

Passive and active buckling enhancement and vibrations control were the subject of interest by many researchers. Different analytical models concerning extension, bending and shear deformations induced via strain actuators bonded and embedded in onedimensional structures, specific characteristics and implications of piezo actuators practical use were discussed in [1]. Experimental investigation of active buckling control and theoretical discussion of the pin-ended composite carbon-epoxy column were performed by Thompson and Loughan [2]. It was showed that imperfections and geometrical nature significantly affect the system's critical buckling load. Ultimate load was significantly lower than the critical buckling load determined for the ideal column. Via the active control the out of plane deflection could be completely removed, restoring the column behaviour under compression. Despite the fact that the ultimate load was increased, its value was still lower than the critical buckling load determined for the ideal column. Different experimental studies in order to obtain optimum thickness of piezoelectric element for cantilever column were presented in [3]. Faria proposed another way of enhancing the buckling capacity of composite columns [4]. Author presented that stress stiffening effect have significant effect when both column ends have longitudinal displacements fixed. On the basis of above statement in-plane axial residual force is generated, which counteract the effects of a compressive force resulting from prescribed support displacement. Thus, fundamental beam buckling resistance may be considerably altered. Broader literature overview and mathematical models concerning buckling of structural members may be found in [5].

Two significant papers concerning the effect of piezo actuation on static and dynamic behaviour of the beams were elaborated by Faria [4] and Zehetner with Irschik [6]. Faria [4] demonstrated that determination of buckling in composite beams composed of a host beam and bonded piezoelectric patches with the use of pin-force model should have been used with extreme caution. Despite that pin-force model may be used to correctly predict the stress stiffening effect. Zehetner and Irschik [6] had also announced that assumptions taken by other authors concerning inextensible beam axis, the absence of longitudinal displacement under applied load and piezoelectric axial actuation were inadmissible and led to incorrect conclusions. Static and dynamic behaviour of multilayer beams with piezoelectric bending actuators were extensively discussed in [7]. Free vibration eigenvalue problem of thin composite plates was the subject of interest of Hernandes et al. [8], who formulated the problem with finite element method on the basis of Reissner-Mindlin theory including non-linear strain-displacement relations. It was shown that the stress stiffening effect depends on several factors i.e. material properties, placement of piezo actuators, boundary conditions and model geometry. Moreover, via the numerical analysis the effect of in-plane stress on free vibrations even in unconstrained plates was revealed. Vibrations control in composite plates were also discussed in [9-11]. Piezoelectric actuation influence on the flexural vibrations of sandwich beam was discussed in [12]. It was stated that the arrangement of piezoelectric material on the host beam have significant influence on the dynamics characteristics of studied system. Additionally, the relationship between mode shape distribution of the electric potential in the piezoelectric layer in the longitudinal direction and beam curvature, which depends on adopted boundary conditions was presented.

The main purpose of this work is to investigate the influence of piezoelectric actuation in stepped beam on buckling critical load and linear frequency. System's inhomogeneity results from a pair piezoelectric patches, perfectly bonded to the host beam on its top and bottom surface. Three different beam ends that support, eliminating longitudinal displacements are taken into considerations. In the system load is applied as a prescribed 
displacement of one of the beam end support. This type of loading can be also represented with temperature field applied to the beam structure, which with both ends fixed in longitudinal direction result in axial internal force occurrence. In order to enhance buckling and alter the linear vibrations in-plane axial compressive or tensile piezo force is induced, depending on the electric field vector.

\section{Problem formulation}

The analysed scheme composed of a host beam with both ends pinned and two piezoelectric patches mounted on beam's top and bottom surface is presented in Fig. 1.

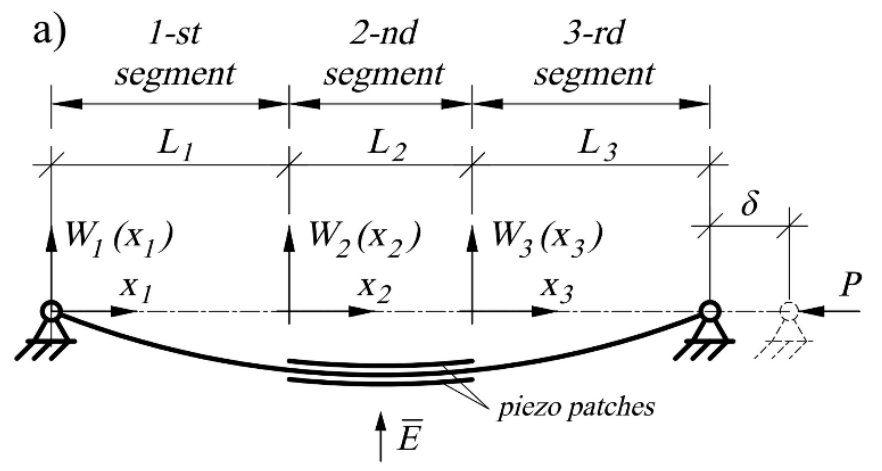

b)

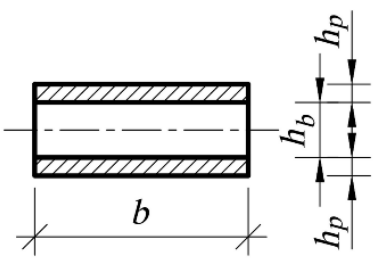

Fig. 1. Scheme of the pinned-pinned beam with two piezoelectric patches symmetrically bonded in the middle segment (a), cross section of the analysed beam (b).

In the study three different beam end supports preventing longitudinal displacements are taken into considerations: both ends clamped, both ends pinned and clamped-pinned. Presented in Fig. 1b cross-section of the beam have following dimensions i.e. exactly the same width of the host beam and piezo patches equal $20 \mathrm{~mm}$, beam height equal $3 \mathrm{~mm}$ and each piezo layer equal $0.5 \mathrm{~mm}$, respectively. It is assumed that piezo elements are perfectly bonded to the beam at the entire length, and adhesive layer thickness have insignificant influence on obtained results. Piezosegment located in the system divides beam symmetrically i.e. first and third segment have same length value. Beam is made of homogeneous, elastic isotropic aluminium material, whereas for the piezo patches homogeneous, elastic, transversely isotropic P41 material is assumed. On the basis of manufacturer [13] piezo elements Young's modulus is $E_{p}=83.33 \mathrm{GPa}$, density $\rho=7450$ $\mathrm{kg} / \mathrm{m}^{3}$, whereas beam Young's modulus is assumed as $70 \mathrm{GPa}$ and density $\rho=2700 \mathrm{~kg} / \mathrm{m}^{3}$, respectively. In the system presented in Fig. 1a, load is applied via the prescribed one of the supports displacement and before the load application the beam is assumed to be rectilinear. Electric field applied to the piezo patches is exactly the same for the top and bottom one. Thus in-plane axial compressive or tensile piezo force can be induced. On the basis of derivation of residual force equation along the stepped beam with $n$-pairs of piezoelectric actuators presented in [14] for three segmented system one obtains:

$$
F_{r}=F\left[1+\eta\left(\frac{L}{L_{2}}-1\right)\right]^{-1}
$$

where: $\mathrm{F}=-2 b e_{31} V$ is the piezoelectric force, which depends on piezoceramic patches width $b, e_{31}$ constant describing piezo material and the applied voltage $V, \eta$ denotes the 
relation of the piezosegment axial stiffness to that of the beam, $L$ is the overall beam length and $L_{2}$ is the length of piezosegment.

The non-dimensional governing equation for $i$-th segment beam with the residual force term for stability and vibrations problem can be expressed as:

- stability problem

$$
\frac{\partial^{4} w_{i}\left(\xi_{i}, \tau\right)}{\partial \xi_{i}{ }^{4}} \pm \varphi_{i} p^{2} \frac{\partial^{2} w_{i}\left(\xi_{i}, \tau\right)}{\partial \xi_{i}{ }^{2}}=0, \quad p^{2}=\left(f_{r}^{2}+p_{u}^{2}\right), \quad \text { for } \quad \mathrm{i}=1,2,3
$$

- vibrations frequency

$$
\frac{\partial^{4} w_{i}\left(\xi_{i}, \tau\right)}{\partial \xi_{i}{ }^{4}} \pm \varphi_{i} f_{r}^{2} \frac{\partial^{2} w_{i}\left(\xi_{i}, \tau\right)}{\partial \xi_{i}{ }^{2}}+\mu_{i} \omega^{2} \frac{\partial^{2} w_{i}\left(\xi_{i}, \tau\right)}{\partial \tau^{2}}=0, \quad \text { for } \quad \mathrm{i}=1,2,3
$$

where the dimensionless parameters are defined through substitutions:

$$
\begin{gathered}
w_{i}\left(\xi_{i}, \tau\right)=\frac{W_{i}\left(x_{i}, t\right)}{L}, \quad \xi_{i}=\frac{x_{i}}{L}, \tau=\Omega t, \quad l_{i}=\frac{L_{i}}{L}, \quad \varphi_{i}=\left(1+r_{m}\right)^{-\frac{1}{2}\left(j^{2 i}+1\right)}, \\
r_{m}=\frac{E_{p} I_{p}}{E_{b} I_{b}}, j=\sqrt{-1}, \quad f_{r}^{2}=\frac{F_{r} L^{2}}{E_{b} I_{b}}, \quad p_{u}^{2}=\frac{P L^{2}}{E_{b} J_{b}}, \quad \mu_{i}=\left(\frac{\alpha_{1}+(\eta-1) \alpha_{2}}{\alpha_{1}\left(1+r_{m}\right)}\right)^{\frac{1}{2}\left(j^{2 i}+1\right)}, \\
\alpha_{1}=\frac{E_{p}}{E_{b}}, \quad \alpha_{2}=\frac{\rho_{p}}{\rho_{b}}, \eta=\frac{E_{b} A_{b}+E_{p} A_{p}}{E_{b} A_{b}}, \quad \omega^{2}=\Omega^{2} L^{4} \frac{\rho_{b} A_{b}}{E_{b} I_{b}}
\end{gathered}
$$

For the substitutions following notation is introduced: $w_{i}$ denotes the transversal displacement, $t$ - time, $E_{p} J_{p}, E_{b} J_{b}$ - piezo element and beam bending stiffness, respectively, $\rho_{p}, \rho_{b}$ - densities of the actuators and beam, respectively, $A_{p}, A_{b}$ - the cross section area of piezopatches and beam, respectively, $\omega$ - natural vibration frequency.

Non-dimensional boundary conditions necessary to obtain the solution of stability and transversal vibrations problem for all analysed schemes are expressed as:

- clamped-clamped system

$$
\left.w_{1}\left(\xi_{1}, \tau\right)\right|_{\xi_{1}=0}=\left.w_{1}^{I}\left(\xi_{1}, \tau\right)\right|_{\xi_{1}=0}=\left.w_{3}\left(\xi_{3}, \tau\right)\right|_{\xi_{3}=0}=\left.w_{3}^{I}\left(\xi_{3}, \tau\right)\right|_{\xi_{1}=0}=0
$$

- clamped-pinned

$$
\left.w_{1}\left(\xi_{1}, \tau\right)\right|_{\xi_{1}=0}=\left.w_{1}^{I}\left(\xi_{1}, \tau\right)\right|_{\xi_{1}=0}=\left.w_{3}\left(\xi_{3}, \tau\right)\right|_{\xi_{3}=0}=\left.w_{3}^{I I}\left(\xi_{3}, \tau\right)\right|_{\xi_{1}=0}=0
$$

- pinned-pinned system

$$
\left.w_{1}\left(\xi_{1}, \tau\right)\right|_{\xi_{1}=0}=\left.w_{1}^{I I}\left(\xi_{1}, \tau\right)\right|_{\xi_{1}=0}=\left.w_{3}\left(\xi_{3}, \tau\right)\right|_{\xi_{3}=0}=\left.w_{3}^{I I}\left(\xi_{3}, \tau\right)\right|_{\xi_{1}=0}=0
$$

where: I and II are the Roman numerals denoting the order of the derivative with respect to the space variable $\xi$.

Continuity boundary conditions which describe the equality of transverse displacements, slopes, bending moments and shear forces between segments are: 


$$
\begin{aligned}
& \left.w_{i}\left(\xi_{i}, \tau\right)\right|_{\xi_{i}=l i}=\left.w_{i+1}\left(\xi_{i+1}, \tau\right)\right|_{\xi_{i+1}=0} \\
& \left.w_{i}^{I}\left(\xi_{i}, \tau\right)\right|_{\xi_{i}=l i}=\left.w_{i+1}^{I}\left(\xi_{i+1}, \tau\right)\right|_{\xi_{i+1}=0} \\
& \left.\left(1+r_{m}\right) \frac{1}{2}\left(j^{2 i}+1\right) w_{i}^{R n}\left(\xi_{i}, \tau\right)\right|_{\xi_{i}=l_{i}}=\left.\left(1+r_{m}\right)^{\frac{1}{2}\left(j^{2(i+1)}+1\right)} w_{i+1}^{R n}\left(\xi_{i+1}, \tau\right)\right|_{\xi_{i+1}=0}, \\
& i=1,2 ; R n=I I, I I I
\end{aligned}
$$

\section{Approximate solutions}

\subsection{Stability problem}

By separating space and time variable with the following formula:

$$
w_{i}\left(\xi_{i}, \tau\right)=w_{i}\left(\xi_{i}\right) \cos \tau
$$

which is introduced to the equations $\left(2_{1}\right)$ one obtains:

$$
\frac{\partial^{4} w_{i}\left(\xi_{i}\right)}{\partial \xi_{i}{ }^{4}} \pm \varphi_{i} p^{2} \frac{\partial^{2} w_{i}\left(\xi_{i}\right)}{\partial \xi_{i}{ }^{2}}=0, \quad p^{2}=\left( \pm f_{r}^{2}+p_{u}^{2}\right), \quad \text { for } \mathrm{i}=1,2,3
$$

The general solution of equation (6), can be demonstrated as follows:

$$
w_{i}\left(\xi_{i}\right)=A_{i} \cos \left(p \xi_{i}\right)+B_{i} \sin \left(p \xi_{i}\right)+C_{i}\left(\xi_{i}\right)+D_{i}, \quad p^{2}=f_{r}^{2}+p_{u}^{2}, \quad i=1,2,3
$$

By substituting equation (7) into the adequate set of boundary conditions $\left(4_{1}-4_{6}\right)$ one obtains a system of twelve homogeneous linear equations with respect to unknown integration constants $A_{i}, B_{i}, C_{i}, D_{i}$ where $i=1,2,3$. Equating to zero the determinant of matrix coefficients of that system one derives a transcendental equation from which the set of successive critical loads can be numerically determined. Each of the buckling modes can be obtained after introduction of a particular eigenvalue into the normalized eigenfunction expressed by equation (7).

\subsection{Vibrations frequency}

Introducing into the governing equation of motion $\left(2_{2}\right)$ the separation of time and space variable formula (5), one obtains:

$$
\begin{gathered}
\frac{\partial^{4} w_{i}\left(\xi_{i}\right)}{\partial \xi_{i}{ }^{4}} \pm \varphi_{i} p^{2} \frac{\partial^{2} w_{i}\left(\xi_{i}\right)}{\partial \xi_{i}^{2}}-\mu_{i} \omega^{2} w_{i}\left(\xi_{i}\right)=0, \\
p^{2}=\left( \pm f_{r}^{2}+p_{u}^{2}\right), \quad \text { for } \mathrm{i}=1,2,3
\end{gathered}
$$

The general solution takes the form:

$$
w_{i}\left(\xi_{i}\right)=A_{i} \cosh \left(\gamma_{1} \xi_{i}\right)+B_{i} \sinh \left(\gamma_{1} \xi_{i}\right)+C_{i} \cosh \left(\gamma_{2} \xi_{i}\right)+D_{i} \sinh \left(\gamma_{2} \xi_{i}\right), \quad i=1,2,3
$$

where coefficients $\gamma_{1}$ and $\gamma_{2}$ are expressed as: 
- in case of compressive force $p^{2}>0$ :

$$
\gamma_{1}=\sqrt{\frac{-\varphi_{i} p^{2}-\sqrt{\varphi_{i} p^{4}+4 \mu_{i} \omega^{2}}}{2}} ; \quad \gamma_{2}=\sqrt{\frac{-\varphi_{i} p^{2}+\sqrt{\varphi_{i} p^{4}+4 \mu_{i} \omega^{2}}}{2}}
$$

- in case of stretching force $p^{2}<0$ :

$$
\gamma_{1}=\sqrt{\frac{\varphi_{i} p^{2}-\sqrt{\varphi_{i} p^{4}+4 \mu_{i} \omega^{2}}}{2}} ; \quad \gamma_{2}=\sqrt{\frac{\varphi_{i} p^{2}+\sqrt{\varphi_{i} p^{4}+4 \mu_{i} \omega^{2}}}{2}}
$$

Once again substituting equation (9) into the adequate set of boundary conditions $\left(4_{1}-\right.$ $4_{6}$ ) twelve homogeneous linear equations with respect to unknown integration constants $A_{i}$, $B_{i}, C_{i}, D_{i}$ where $i=1,2,3$ are obtained. Equating the matrix of the coefficients standing within the unknown integration constants to zero, the solution describing successive natural frequencies is determined.

\section{Numerical results and discussion}

In this chapter particular attention has been dedicated to the buckling enhancement and vibration control depending on the piezosegment length and the electric field vector direction. The piezosegment length in the range of $l_{2} \in(0 ; 1)$ has been investigated. The influence of piezosegment placement and other physical and structural parameters on buckling and natural vibration frequency is presented in $[15,16]$. Knowing that both ends of the beam are constrained against longitudinal displacement, axial tensile or compressive force can be introduced to the system via piezo patches. Thus piezoelectric force parameter $f$ have been assumed to be equal the pinned-pinned beam critical buckling load parameter:

$$
f=\sqrt{\frac{P_{c r} L^{2}}{E_{b} J_{b}}}=\pi
$$

It is worth noting that the residual force (1) can be equal to piezoelectric force only in case, if the piezoceramic actuators covers the entire beam length and axial stiffness of the host beam and actuators is exactly the same, in other cases $f_{r}<f$.

However, all numerical results are going to be presented in non-dimensional form, an analysis of practical model should also be demonstrated. Beam with length of $L=400[\mathrm{~mm}]$ is assumed in order to verify necessary voltage to generate the piezoelectric force. On the basis of the P-41 material and properties provided by the manufacturer [13], a piezoelectric constant of value $d_{31}=6.65 \cdot 10^{-11}[\mathrm{C} / \mathrm{N}]$ has been adopted. The critical buckling load for the pinned-pinned beam on the basis of equation (9) is equal to 194.31 $[\mathrm{N}]$, hence an individual piezoceramic actuator should generate a force equal to 97.16 [N]. On the basis of above, necessary value of the applied electric field should reach the value of 582.95 [V], which is far beyond the piezoceramics depolarisation point equal 1000 [V]. Hence, assumed piezoelectric force can be safely treated as a tool for enhancing buckling and alter the natural vibration frequency. 


\subsection{Buckling enhancement}

In Figs. 2a-2d enhancement ranges of critical buckling force for the clamped-clamped, clamped-pinned and pinned-pinned beam have been presented. In Figs. 2a-2c with the solid line type critical buckling load in the function of piezosegment length without piezo actuation $f=0$ is presented. With the dashed line tensile piezoelectric force influence on buckling is presented, whereas with dash-dot line compressive piezo force, respectively. In the Fig. $2 d$ percentage enhancement of buckling load in the function of piezosegment length is shown.

a)

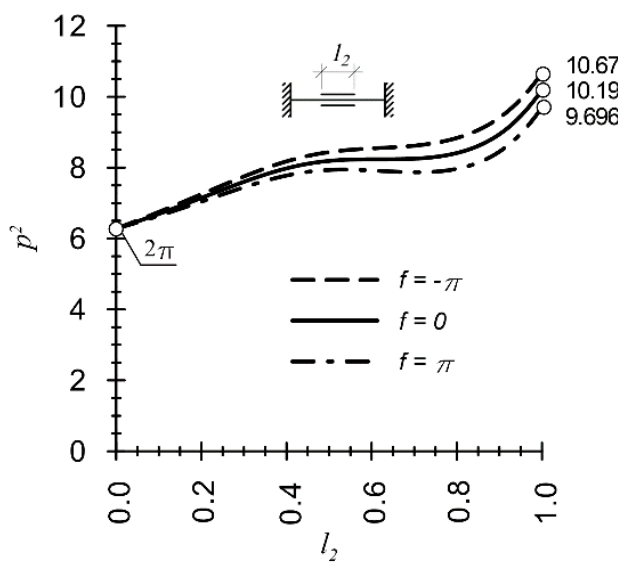

c)

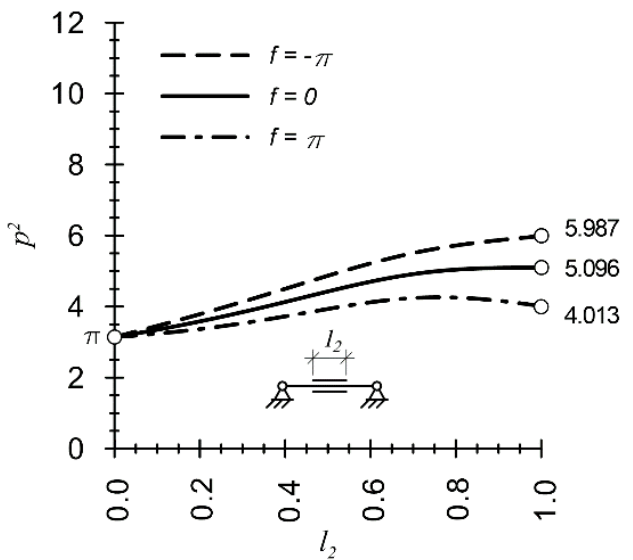

b)

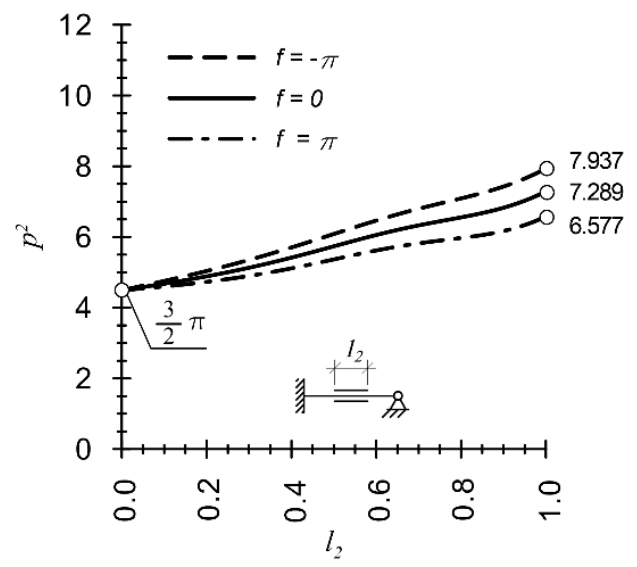

d)

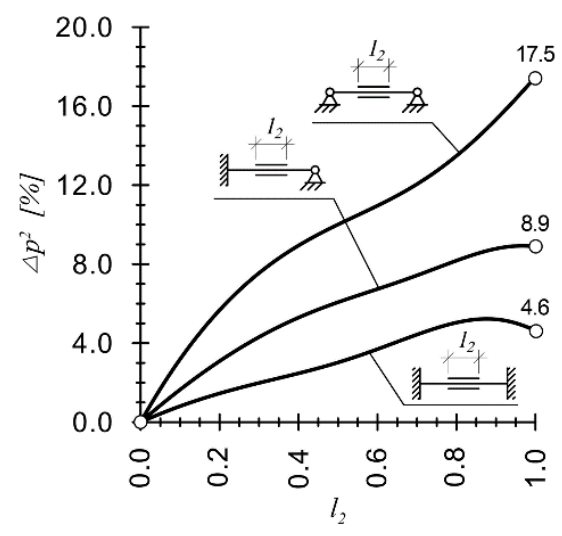

Fig. 2. Modification range of the critical buckling load for different piezosegment length, (a) clamped-clamped beam, (b) clamped-pinned beam, (c) pinned-pinned beam, (d) percentage range of critical buckling load enhancement via axial tensile residual force for different beam ends supports

Comparing results presented in Figs. 2a-2c one can state that regardless of beam ends supports, for systems without piezo actuation, critical buckling load increases together with increased length of the piezosegment. In all discussed systems, the tensile piezoelectric force result in buckling load increase, whereas compressive piezo force acts in opposite way. It is worth noting that for the beam with both ends clamped, the highest percentage influence of piezoelectric actuation (see Fig. 2d) is obtained in case of piezosegment length $l_{2}=0.88$ and is equal $5.23 \%$. Further increase of piezosegment length in discussed beam 
results in decreased effectiveness. For the beam where supporting ends are assumed pinnedpinned (Fig. 2b) and clamped-pinned (Fig. 2c) together with increased piezosegment length, buckling load is increased. It should be noted that the lower rotation stiffness provided by the adopted supports in the system, the stronger effect of the induced axial residual force and wider range of possible buckling force modification is obtained (see Fig. 2d)

\subsection{Vibrations control}

In Figs. 3a-3c non-dimensional natural vibrations frequency is shown for the system with following ends supports: clamped-clamped, clamped-pinned, pinned-pinned, respectively. Once again with solid line system without piezo actuation is presented, dashed line denotes the compressive piezo force, whereas dash-dot line corresponds to the system with tensile axial piezo force induced. In Figs. $4 \mathrm{a}-4 \mathrm{~b}$ percentage modification of natural system frequency for compressive and tensile piezoelectric force is shown for all analysed systems.

a)

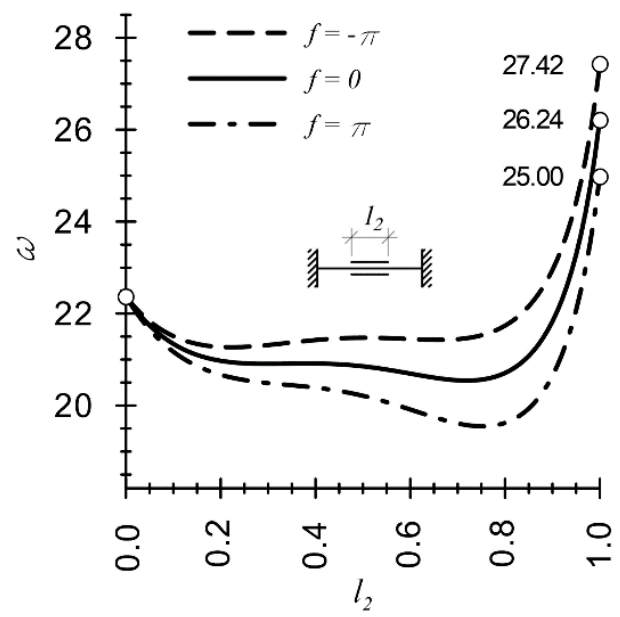

c)

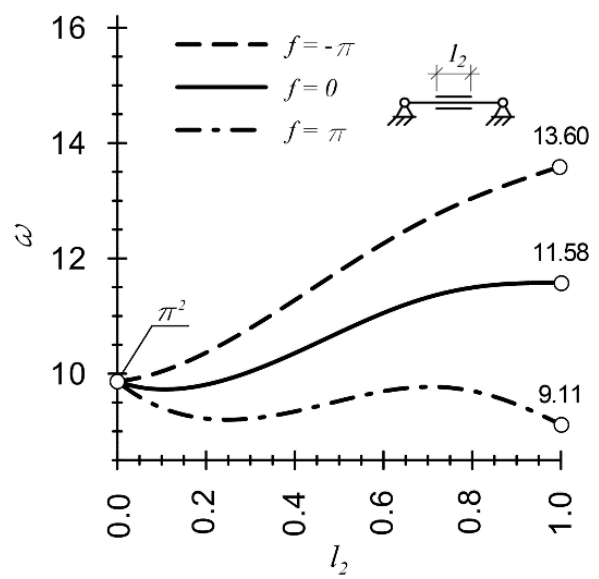

b)

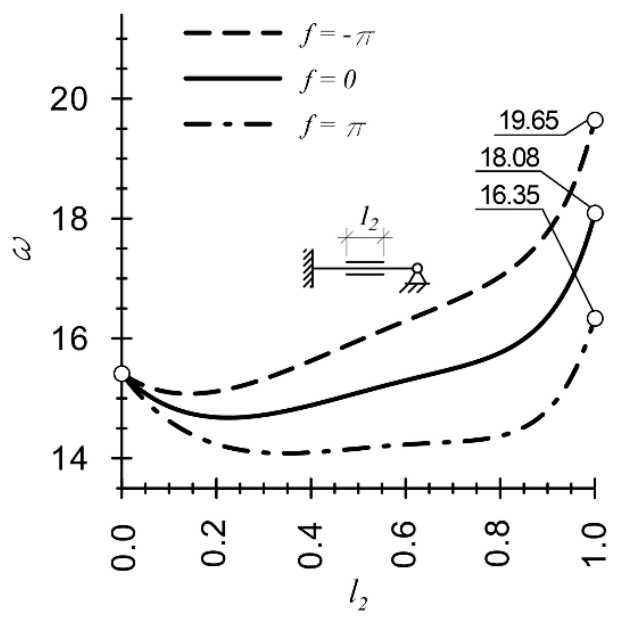

d)

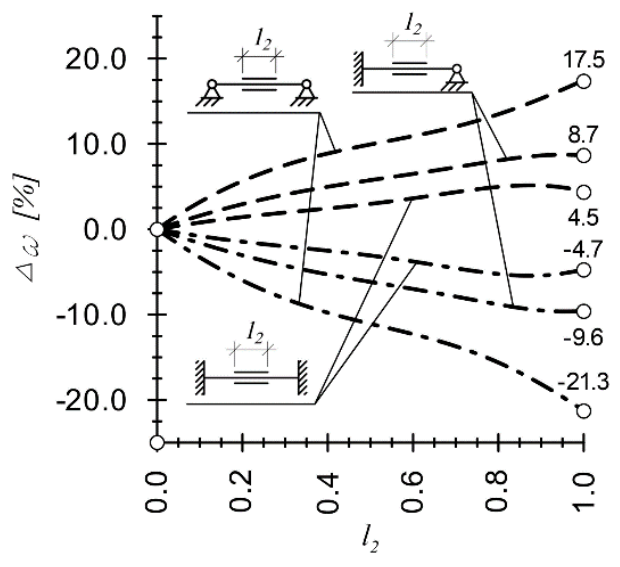

Fig. 3. The first natural frequency in the function of piezosegment length and different values of piezoelectric force induced, (a) clamped-clamped beam, (b) clamped-pinned beam, (c) pinned-pinned beam, (d) percentage range of natural system's frequency modification via piezo actuation for different beam ends supports 
Considering numerical results presented in Fig. 3a-3c one can state that axial tensile piezoelectric force increases the first natural vibration frequency of each system, whereas compressive piezo force diminishes that frequency, respectively. It should be noted that natural frequency curves changes irregularly regardless of system supporting. The reason of that behaviour are: the relation of piezosegment axial stiffness to the host beam stiffness, piezo patches bending stiffness to the beam stiffness and mass distribution per unit length. For the clamped-clamped (Fig. 3a) and clamped-pinned (Fig. 3b) system at small values of piezosegment length, decrease of first natural frequency can be observed, furtherly it starts to increase. Independently of the piezoelectric force value for the clamped-clamped beam (Fig 3a) rapid increase of the first natural frequency can be observed. Similar behaviour may be observed in the clamped-pinned beam (Fig. 3b) in case of piezo patches length greater than 0.85 entire beam length. On the basis of presented curves in Fig. $3 \mathrm{~d}$, again one can state that the widest range of possible first natural frequency modification with the axial tensile and compressive residual force is obtained for the pinned-pinned beam, whereas the narrowest range for clamped-clamped system is obtained, respectively.

\section{Conclusion}

In this study the problem of buckling enhancement and alteration of first natural frequency via the piezo actuation for the system with various type of ends supports preventing longitudinal displacement have been discussed. The priority parameters concerned the support conditions, geometrical parameters and the piezoelectric actuation.

Regardless of the beam ends supporting induce of the axial tensile residual force counteracts the external loading, significantly enhancing the critical buckling force. Moreover, same piezoelectric force results in increased first natural vibration frequency in any analysed system in regard to that system without piezo actuation. Compressive force acts in the opposite manner. Additionally, one can be observed that the lower system's supports rotational resistance the wider range of possible system's buckling and vibrations control is obtained. It is worth noting that the range of possible critical load or natural vibration frequency control depends strongly on piezo patches geometry, relation of piezo axial and bending stiffness to the host beam and maximum allowable voltage avoiding the risk of piezo elements depolarisation. Presented numerical results concern only one of many possible configurations of the system, thus additional research and experimental tests should be provided continuing this study.

\section{References}

1. E. F. Crawley, E. H. Anderson, Detailed models of piezoelectric actuation of beams. Journal of Intelligent Material Systems and Structures 1, 4-25 (2016)

2. S. Thompson, J. Loughan, The active buckling control of some composite column strips using piezoceramic actuators. Composite Structures 32, 59-67 (1995)

3. M. J. Cunningham, D. F. L. Jenkins, W. W. Clegg, M. M. Bakush, Active vibration control and actuation of a small cantilever for applications in scanning probe instruments. Sensors and Actuators A: Physical 50, 147-150 (1995)

4. A. R. de Faria, On buckling enhancement of laminated beams with piezoelectric actuators via stress stiffening. Composite Structures 65, 187-192 (2004)

5. C. M. Wang, C. Y. Wang, J. N. Reddy, Exact solutions for buckling of structural members. (Computational mechanics and applied analysis, CRC Press, 2005) 
6. C. Zehetner, H. Irschik, On the static and dynamic stability of beams with an axial piezoelectric actuation. Smart Structures and Systems 4 (1), 67-84 (2008)

7. R. G. Ballas, Piezoelectric multilayer beam bending actuators: Static and dynamic behaviour and aspects of sensor integration. (Springer, 2007)

8. J. A. Hernandes, S. F. M. de Almeida, A. Nabarrete, Stiffening effect on the free vibrations behaviour of composite plates with PZC actuators. Composite Structures 49 (1), 55-63 (2000)

9. K. Narwal, D. Chhabra, Analysis of simple supported plate for active vibration control with piezoelectric sensors and actuators. Journal of Mechanical and Civil Engineering 1 (1), 26-39 (2012)

10. R. C. Batra, X. Q. Liang, The vibration of a rectangular laminated elastic plate with embedded piezoelectric sensors and actuators. Computers \& Structures 63 (2), 203216 (1997)

11. R. C. Batra, X. Q. Liang, J. S. Yang, Shape control of vibrating simply supported rectangular plates, AIAAJ Journal 34 (1), 116-122 (1996)

12. Q. Wang, S. T. Quek, Flexural vibration analysis of sandwich beam coupled with piezoelectric actuator. Smart Materials and Structures 9 (1), 103-109 (2000)

13. Piezoelectric material properties given by manufacturer. www.annon-piezo.com/pztmaterials.asp (date accessed: 20 July 2017)

14. J. Przybylski, Static and dynamic behaviour of slender mechanical systems with integrated piezoceramic elements. (Wydawnictwo Politechniki Częstochowskiej, Częstochowa, 2012) [in Polish]

15. K. Kuliński, J. Przybylski, Lateral displacements of sandwich beams laying on elastic foundation. Modelowanie Inżynierskie 42, 33-40 (2015) [in Polish]

16. K. Kuliński, J. Przybylski, Buckling of stepped beams resting on an elastic foundation. Journal of Applied Mathematics and Computational Mechanics 14 (4), 115-126 (2015) 